\title{
MOVING HORIZON ESTIMATION FOR AN INDUSTRIAL GAS PHASE POLYMERIZATION REACTOR
}

\author{
Jasmeer Ramlal* Kenneth V. Allsford ${ }^{* *}$ \\ John D. Hedengren ${ }^{* * *, 1}$ \\ * Sasol Polymers, 56 Grosvenor Road, Bryanston, \\ Randburg, South Africa 2125 \\ ** Honeywell International, 1250 W Sam Houston Pkwy, \\ Houston, Texas, USA 77042 \\ *** APMonitor.com, 1518 Beechurst Ct, Houston, TX, \\ USA 77062
}

\begin{abstract}
A large-scale set of differential and algebraic equations (DAEs) is used to model and control an industrial gas phase polymerization reactor. Moving horizon estimation, preceding the nonlinear control optimization, provides an estimate of the current states and unmeasured disturbances. MHE is compared to Implicit Dynamic Feedback (IDF ${ }^{\mathrm{TM}}$ ) *. With MHE, there is improved estimation of unmodeled disturbances in the UNIPOL ${ }^{\mathrm{TM}} * *$ polyethylene plant. The polymerization model is converted to algebraic equations by orthogonal collocation and solved with the MHE objective function in a simultaneous optimization. NOVA ${ }^{\mathrm{TM}}$, an activeset sparse NLP solver, is used to converge the problem that has 46,870 variables. This large, sparse optimization problem is initiated every 5 minutes to update the model as new plant measurements become available and prior to the control optimization. The same plant model is used for nonlinear model predictive control (MPC) with 10 manipulated variables (MVs) and 26 controlled variables (CVs). In this case, MHE improves the control by better estimating reactor compositions of hydrogen, the condensing agent, and other measured state variables.

* Unless otherwise indicated, all trademarks listed in this paper are of Honeywell International

** The UNIPOL ${ }^{\mathrm{TM}}$ technology is licensed by Univation, a joint venture between ExxonMobil and Dow.
\end{abstract}

Keywords: Moving horizon estimation, nonlinear model predictive control, polymerization modeling

\section{INTRODUCTION}

Unmodeled disturbances or features of a physical process pose a limitation, in particular, for model-based control. Before optimal manipulated

1 To who correspondence should be addressed:

E-mail: john@hedengren.net variable (MV) moves can be computed, the current model states must reasonably match the measurements from the actual process. Qin and Badgwell (Qin and Badgwell, 2000) surveyed the current state of nonlinear model predictive control (MPC) applications. According to the survey, the most popular feedback techniques at the time were constant and integrating output bias distur- 
bances. Froisy concluded that multivariable state estimation is a key enabler that allows linear MPC to reject disturbances more effectively than a well tuned PID controller (Froisy, 2006).

State estimation of real systems may include changing measurement frequencies and missing measurements, multiple measurements at different sampling frequencies, measurement delay, large-scale nonlinear models, and constraints. $\mathrm{MHE}$, as a part of nonlinear dynamic data reconciliation (Soderstrom et al., 2000), is flexible to deal with these scenarios (Moraal and Grizzle, 1995). Liebman et al. was the first to propose a simultaneous solution approach for MHE where the differential equations are transformed into algebraic equations through orthogonal collocation on finite elements (Liebman et al., 1992). Later, Albuquerque and Biegler improved the solution approach by exploiting the MHE SQP structure to achieve linear computational scaling with horizon length (Albuquerque and Biegler, 1995). Further computation improvement was proposed by Hedengren et al. in an explicit solution to a simplified nonlinear MHE problem (Hedengren and Edgar, 2006).

Many of the papers on MHE discuss novel theoretical aspects but do not address some practical issues for industrial applications. The contribution of this work is to discuss an industrial application of MHE including practical issues in implementation and discuss how $\mathrm{IDF}^{\mathrm{TM}}$ and $\mathrm{MHE}$ can be used with nonlinear MPC. Additional results and discussion can be found in a similar paper on the same application(Hedengren et al., 2007). Other relevant papers are from ExxonMobil Chemical Company on MHE for diluent inventory tracking (Soderstrom et al., 2000) and a liquid phase polymerization reactor (Russo and Young, 1999). Together, these studies show the success of MHE as applied to models of industrial processes.

\section{FEEDBACK STRATEGIES}

MHE is compared with Implicit Dynamic Feedback $\left(\mathrm{IDF}^{\mathrm{TM}}\right)$ in this paper. These feedback technologies are available as options in Honeywell's ProfitNLC ${ }^{\mathrm{TM}}$ product to incorporate model feedback of measured states.

\subsection{Implicit Dynamic Feedback (IDF $\left.{ }^{T M}\right)$}

The IDF ${ }^{\mathrm{TM}}$ formulation is a feedback strategy that estimates disturbances related to the predictions of the measured state variables. $\mathrm{IDF}^{\mathrm{TM}}$ is a pairing of these measured states with unmeasured disturbance variables. The disturbance variable is adjusted to reduce model discrepancies with process measurements. The $\mathrm{IDF}^{\mathrm{TM}}$ formulation is compact enough as to be incorporated simultaneously with the control problem within one history interval.

$\mathrm{IDF}^{\mathrm{TM}}$ is a proportional integral (PI) controller that is integrated with the process model. The PI controller input is the error between the measured state $\left(y_{s}\right)$ and model state $\left(y_{m}\right)$. The output is an unmeasured disturbance variable $(d)$ of the model. This disturbance variable is adjusted proportional to the current and integrated measurement error.

$$
d=K\left(y_{s}-y_{m}\right)+\frac{K}{\tau_{I}} \int_{t=0}^{T}\left(y_{s}-y_{m}\right) d t
$$

The tuning parameters for $\operatorname{IDF}^{\mathrm{TM}}$ are $K$ and $\tau_{I}$, the same as a PI controller. Using a large value of $\tau_{I}$ and small $K$ has the affect of heavily filtering the error term for feedback. In this case the algorithm will take longer to match the plant. Using these tuning parameters and knowing the quality and types of measurements enables trading off of "speed of tracking the plant" versus "stability concerns". IDF ${ }^{\mathrm{TM}}$ has been successfully used for seven years to provide on-line estimation measurement biases, catalyst activities, kinetic parameter adjustment factors and heat transfer coefficients. However, $\mathrm{IDF}^{\mathrm{TM}}$ limitations are a limitation to a past horizon length of one, requirement of equal number of disturbance variables and measurements, and an inability to handle disturbance variable constraints.

\subsection{Moving Horizon Estimation}

The MHE objective function is posed as a minimization of L2-norm error to reconcile the model with measured values. Similar to $\mathrm{IDF}^{\mathrm{TM}}$, the disturbance variables $(d)$ are the only degrees of freedom to adjust model predictions to match measurements.

$$
\begin{aligned}
& \min _{d} \Phi=\left\|\frac{y_{s}-y_{m}}{y_{m}}\right\|_{Q_{y}}^{2}+\|d-\hat{d}\|_{Q_{d}}^{2} \\
& \text { s.t. } \quad 0=f(\dot{x}, x, u, d) \\
& \quad y_{s}=g(x, u, d) \\
& \quad a \geq h(x, d) \geq b
\end{aligned}
$$

where subscript $s$ refers to sample values, and subscript $m$ refers to model values, $\Phi$ is the objective function value, $y_{s}$ is a vector of measurements at all nodes in the orthogonal collocation structure $\left(y_{s, 0}, \ldots, y_{s, n}\right)^{T}, y_{m}$ is a vector of model values at the sampling times $\left(y_{m, 0}, \ldots, y_{m, n}\right)^{T}, Q_{y}$ is optimally the inverse of the measurement error covariance, $f$ is a vector of model equation residuals, $x$ represents the model states, $u$ is the vector of model inputs, $d$ is the vector of model parameters 
or unmeasured disturbances, $\hat{d}$ is the vector of previous unmeasured disturbances, $Q_{d}$ is a matrix for the weight on changes of disturbance variables, $g$ is an output function, $h$ is an inequality constraint function, and $a$ and $b$ are lower and upper limits, respectively.

Note that the full MHE problem would allow violation of the state constraints and estimation of the initial states. In such a formulation, state continuity constraints are relaxed and violations are penalized in the objective function. The optimization problem found in Equation 2 does not allow state transition error because the state equations are exactly satisfied at a converged solution (Binder et al., 2001). State estimation is accomplished by adjusting past values of disturbance variables. This approach greatly reduces the number of optimization degrees of freedom while converging to a solution that is consistent with the measurements.

With a long extended prediction horizon of the controller, it is important to have a reliable future prediction of the disturbance. For this reason, the disturbance value is held constant over the MHE horizon. This single disturbance value is then projected into the future for the control optimization. Allowing the disturbance value to vary from node to node did not produce a reliable future prediction. Additionally, the penalization for changes in the disturbance acts to improve the reliability of the predicted disturbance.

A drawback to MHE implementation can be summarized as "industrial inertia" due to configuration and compute time concerns.

\section{POLYMERIZATION MODELING}

The polymerization modeling discussed here is used directly in the nonlinear control application (see Young (Young, 2001)). The gas phase polymerization reactor and plant are modeled with the Polymer Dynamic Modeling System (PDMS ${ }^{\mathrm{TM}}$ ). The model consists of heat, mass, and species balances with a kinetic mechanism for the polymerization reactor. The kinetic model and method of moments for the polymer properties is based on the work by Ray (Ray, 1972). In addition to the fluidized bed reactor model, the flowsheet includes a compressor, heat exchanger, vent stream, feed streams, polymer takeoff line for degassing, and dynamic models of the base regulatory control system.

The steady state model, $0=f(\dot{x}=0, x, u, d)$, has 1927 state variables, 764 fixed model inputs, and 1 complementarity condition to account for the variable condensed phase of the heat exchanger. For nonlinear MPC, the model is solved over a time horizon with $\dot{x} \neq 0$. There are a total of 10 MVs and $26 \mathrm{CVs}$, including some pass-through CVs (MVs with CV tuning). The MVs are the feed flows to the reactor, reactor temperature, and vent to the monomer recovery unit. The CVs include polymer properties, reactor pressure, reactor ethylene partial pressure, and process constraints. The following discussion focuses on feedback formulation in relation to improving the prediction of the effect of MV changes on the dynamic and final values for the CVs. A diagram of the flowsheet model is shown in Figure 1.

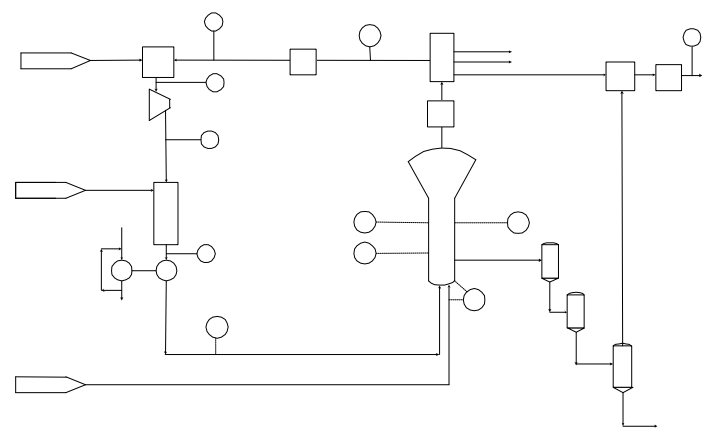

Fig. 1. Flowsheet of the polyethylene plant model.

$\mathrm{IDF}^{\mathrm{TM}}$ is implemented within the application as a disturbance estimator for disturbances not included in MHE and as a backup for MHE estimation of disturbances. There are $27 \mathrm{IDF}^{\mathrm{TM}}$ pairs that couple measured state errors to model disturbance variables. IDF ${ }^{\mathrm{TM}}$ actively manages 19 disturbances, with backup configuration for the 8 MHE disturbances. Note that the number of $\mathrm{IDF}^{\mathrm{TM}}$ pairs is larger than the number of CVs. This arises as some CVs are duplicated as measured states and matched by adjusting input disturbances, which are tuned less aggressively. Aggressive tuning on output biases is generally incorporated for CVs explicitly exposed to the controller. Also, note that the input disturbances are abstractly set within the model to minimize the model dynamics with respect to the model measured state. This is done to also allow for more aggressive tuning of $\mathrm{IDF}^{\mathrm{TM}}$.

Due to the choice of MVs, many of the unmeasured input disturbances are ficticious flows to and from the cycle gas loop to enable matching of composition and pressure. This includes input disturbance flows for both inert components and reacting components. The inert compositions are nearly integrating variables because they are not consumed in the reaction. Practically, hydrogen composition has some characteristics of these inerts components as it takes significant time to react away given the concentration in the cycle gas. The dynamics for inerts and hydrogen described here are less well-suited for best performance of $\mathrm{IDF}^{\mathrm{TM}}$. In addition, there are some known process phenomena that are not encapsulated within the 
originally implemented model. These phenomena relate to adsorption and desorption within the polymer bed of heavy hydrocarbon components. Taking these issues into consideration, one could expect that the $\mathrm{IDF}^{\mathrm{TM}}$ feedback on the relevant input disturbances may lead to oscillation. As such, 8 of these input disturbances were defined to be updated by MHE.

As configured, the MHE optimization problem contributes an objective function and 8 degrees of freedom for the 8 disturbance variables referenced in Table 1. These disturbances are configured to move only on the first collocation point of the first interval in the horizon. Because MHE does not require one to one pairing of disturbance variables and measured states, additional measurements may be included in the objective function without adding disturbance variables. These measured states are comonomer (comonomer A and/or comonomer B) to monomer (ethylene) mole ratios in the reactor. For control of properties, it is more important to match this ratio rather than the absolute mole fraction of either. This is reflected in the higher weighting given to these mole ratios.

Table 1. IDF $^{\mathrm{TM}}$ Pairing and Tuning

\begin{tabular}{llll}
\hline Measured State & Disturbance & $K$ & $\begin{array}{l}\tau_{I} \\
(\text { min })\end{array}$ \\
\hline C2= mol\% & C2= Feed Bias & 3 & 5 \\
Com A mol\% & Com A Feed Bias & 2 & 5 \\
Com B mol\% & Com B Feed Bias & 4 & 5 \\
Condensing & Condensing Agent & 0.25 & 5 \\
Agent mol\% & Feed Bias & & \\
Hydrogen mol\% & Hydrogen & 1 & 5 \\
& Feed Bias & & \\
Pressure & Vent Flow Bias & 50 & 5 \\
Prod Rate & Cat Activity & 0.0001 & 10 \\
Cooler Temp & Heat Bias & 0.5 & 10 \\
\hline
\end{tabular}

The tuning parameters were set through the following strategy. First, the relative importance of the measured states was defined and weights set such that the reference weight was 1 . All tuning matrices are diagonal in this application. Weights on the measured states were increased across the board, for selected intervals of the horizon. This weighting reflected the desire to have current states match the currently measured values more closely. Then, typical steady state gains from the model were used to determine initial values for the regularization weights (input weights) on movement of disturbances with the intention of restricting moves compared to no weight. The predictions were monitored each day and the regularization weights adjusted. A half/double rule was used to change the regularization weights and influence the relative movement of the disturbances. After 3 days of online tuning, performance would be judged optimal for the selected horizon. Horizons of 30,50 , and 70 minutes were evaluated and a horizon of 50 minutes was selected. For the 30 minute horizon, there was judged to be unnecessary movement of the disturbances. The tuning parameters or weights on the squared measurement errors and disturbance regularization weights are shown in Table 2.

Table 2. MHE Tuning

\begin{tabular}{lc}
\hline Measured State & Weight \\
\hline Rx Ethylene mol\% & 1.0 \\
Rx Comonomer A mol\% & 0.1 \\
Rx Comonomer B mol\% & 0.1 \\
Rx Condensing Agent mol\% & 1.0 \\
Rx Hydrogen mol\% & 0.1 \\
Rx Pressure & 4.0 \\
Production Rate & 1.0 \\
Cooler Temp & 1.0 \\
Rx ComA/C2 Mole Ratio & 4.0 \\
Rx ComB/C2 Mole Ratio & 4.0 \\
\hline & \\
\hline Input Disturbance & Weight \\
\hline Ethylene Feed Bias & $\frac{1}{200}$ \\
Comonomer A Feed Bias & $\frac{1}{15}$ \\
Condensing Agent Feed Bias & $\frac{1}{7}$ \\
Comonomer B Feed Bias & $\frac{1}{15}$ \\
Hydrogen Feed Bias & $\frac{1}{40}$ \\
Catalyst Activity & $\frac{1}{3}$ \\
Heat Loss Bias & $\frac{1}{50}$ \\
Vent Bias & $\frac{1}{10000}$ \\
\hline
\end{tabular}

The MHE problem typically solves in 2-3 iterations and in under 30 seconds of CPU time on a 3.4 $\mathrm{GHz} \mathrm{PC}$ computer. Once converged, the estimate of the current states and disturbance variables are transferred as initial conditions for the control optimization. In the event that MHE does not converge or converges with an infeasible solution, $\mathrm{IDF}^{\mathrm{TM}}$ automatically initiates.

\section{RESULTS}

These results are from testing performed on an industrial gas phase polymerization plant with on-line process measurements, occasionally corrupt or missing data, and polymer grade and production rate transitions. The process model is run real-time in parallel to the plant, but no optimal control results are transferred to the process (open loop control during model validation prior to controller commissioning). The comparison is between the two modes of feedback noted in Section 3 with one mode exclusive of MHE and the other mode with $\mathrm{IDF}^{\mathrm{TM}}$ and MHE. The $\mathrm{IDF}^{\mathrm{TM}}$ and MHE results are shown for production rate (Figures 2 and 3 ) and reactor hydrogen mole fraction (Figures 4 and 5). All results are normalized to the average measured values for the $\mathrm{IDF}^{\mathrm{TM}}$ period. Both $\mathrm{IDF}^{\mathrm{TM}}$ and MHE periods are shown for 2 days of plant testing, with different time periods for each. A better comparison would be against the same plant data, but performance indicators can still be compared with these results. 

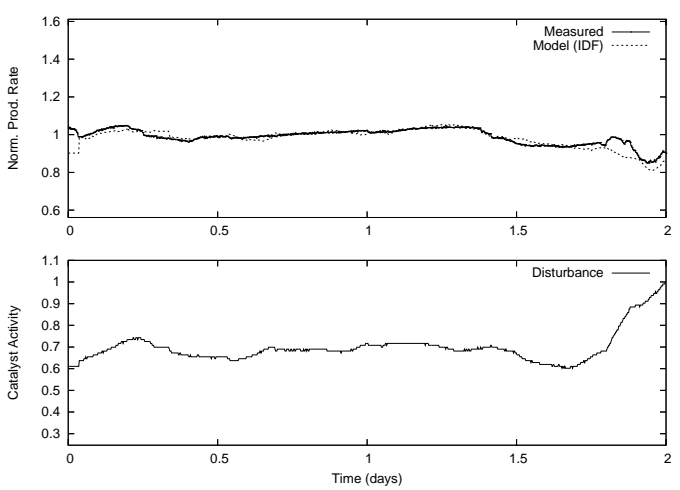

Fig. 2. $\mathrm{IDF}^{\mathrm{TM}}$ for production rate.
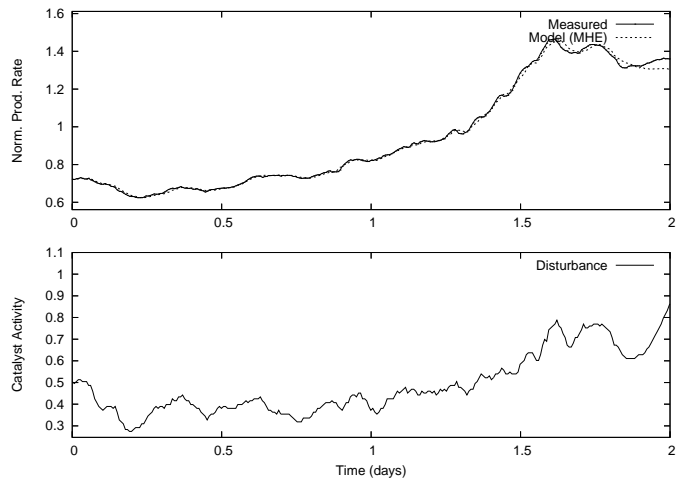

Fig. 3. MHE for production rate.

Two performance indicators for the feedback techniques are (1) Normalized variance of the model error and (2) Variance of the disturbance variable. As a generalization, each indicator is a separate quantitative measure of the feedback effectiveness. Indicator \#1 displays the estimator's ability to track the process (see Table 3). Indicator \#2 displays the ability to track the process without aggressive movement of the disturbance variables (see Table 4). The feedback can be tuned aggressively to improve indicator \#1. However, aggressive feedback will increase indicator \#2 and lead to poor future predictions in the subsequent control problem. Indicator \#1 and \#2 are performance measures for state and disturbance tracking, respectively.

Table 3. Normalized Standard Deviation of Model Error for the Data Shown in Figures 2 to 5

\begin{tabular}{llll}
\hline & IDF $^{\text {TM }}$ & MHE & Improved \\
\hline Production Rate & 0.0275 & 0.0113 & $143 \%$ \\
Pressure & 0.0083 & 0.0019 & $332 \%$ \\
Ethylene mol\% & 0.0059 & 0.0029 & $108 \%$ \\
Hydrogen mol\% & 0.1034 & 0.0026 & $3909 \%$ \\
Condensing & 0.0489 & 0.0014 & $3288 \%$ \\
Agent mol\% & & & \\
\hline
\end{tabular}

The performance indicators for MHE are better than for IDF ${ }^{\mathrm{TM}}$ with the exception of indicator \#2 for catalyst activity. It is speculated that this is due to the production rate increase that occurred during MHE testing, but not during IDF testing. During this time, there was an unmodeled shift in
Table 4. Disturbance Variance for the Data Period in Figures 2 to 5

\begin{tabular}{llll}
\hline Disturbance & IDF $^{\text {TM }}$ & MHE & Improved \\
\hline Cat Activity & 0.08 & 0.14 & $-43 \%$ \\
Vent Bias & 278.53 & 168.24 & $66 \%$ \\
C2= Feed Bias & 5.28 & 4.47 & $18 \%$ \\
H2 Feed Bias & 2.36 & 0.52 & $354 \%$ \\
Condensing Agent & 2.63 & 0.41 & $537 \%$ \\
Feed Bias & & & \\
\hline
\end{tabular}

the production rate that was adjusted by catalyst activity.

The improvements evident for the condensing agent and hydrogen are consistent with the discussion in Section 3. Hydrogen is a chain transfer agent that shortens polymer chains, making less viscous polymer. Roughly one hydrogen molecule is consumed for every polymer chain produced. The consumption rate of hydrogen is small compared with the total amount present in the recirculating reactor gas. This leads to integrating behavior of the disturbance variable. The improved estimation of hydrogen and the condensing agent suggests that MHE is particularly suited for disturbance variables that have integrating effect on the measured state (see Figures 4 and 5).
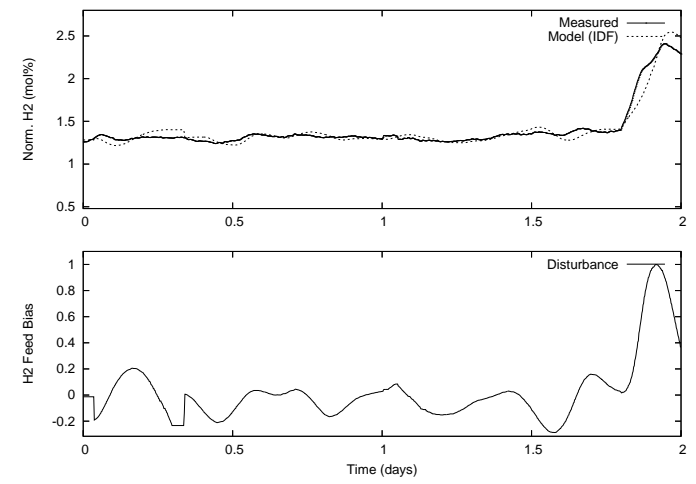

Fig. 4. $\mathrm{IDF}^{\mathrm{TM}}$ for reactor hydrogen mole fraction.
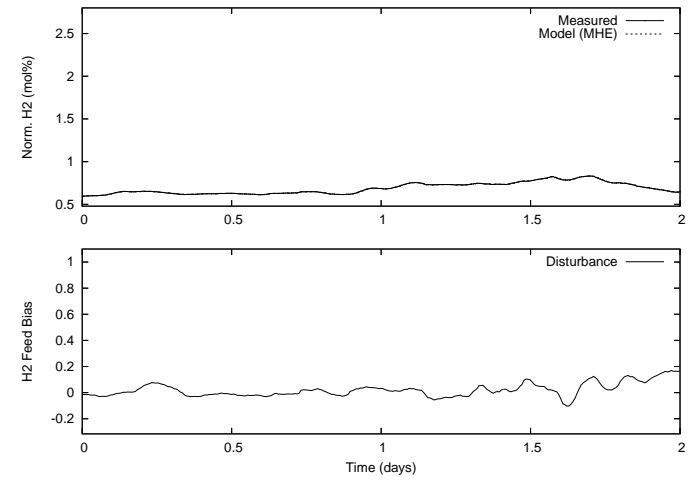

Fig. 5. MHE for reactor hydrogen mole fraction.

\section{CONCLUSIONS}

MHE facilitates improved state estimation compared to $\mathrm{IDF}^{\mathrm{TM}}$ only. There is reduced variability 
of model error and generally reduced movement of disturbance variables. In fact, the project team associated the use of MHE with enabling the use of feed flows as MVs for this control problem. This benefit was gained by improving the model predictions without improving the modeling of the unmeasured disturbances to hydrogen and the condensing agent. MHE was able to readjust past model predictions over a longer horizon, leading to improved current states.

Besides better tracking of the process with less variability in prediction error, a major driving force for MHE application is the use of less sophisticated models for predictive analysis (in this case, nonlinear MPC). MHE enables a simpler model to be used with an improved selection of MVs and additional CVs for an improved control application. Marquardt stated that the major bottleneck to successful nonlinear MPC is the lack of reliable first-principles or hybrid models (Marquardt, 2001). With MHE this bottleneck is reduced by allowing simpler models to be used in more advanced applications. Future focus will center on a more complete MHE formulation for industrial problems.

\section{ACKNOWLEDGMENTS}

The authors would like to gratefully acknowledge the funding and support from SASOL, South Africa, contributions from Dr. Jeff Renfro, and support from Honeywell management.

\section{REFERENCES}

Albuquerque, J.S. and L.T. Biegler (1995). Decomposition algorithms for on-line estimation with nonlinear models. Computers and Chemical Engineering 19(10), 1031-1039.

Binder, T., L. Blank, H.G. Bock, R. Burlisch, W. Dahmen, M. Diehl, T. Kronseder, W. Marquardt, J.P. Schlöder and O.v. Stryk (2001). Online Optimization of Large Scale Systems. Chap. Introduction to model based optimization of chemical processes on moving horizons, pp. 295-339. Springer-Verlag Berlin Heidelberg.

Froisy, J.B. (2006). Model predictive control building a bridge between theory and practice. In: Proc. of the CPC VII. Lake Louise, Alberta, Canada.

Hedengren, J.D. and T.F. Edgar (2006). Moving horizon estimation - the explicit solution. In: Proc. of the CPC VII. Lake Louise, Alberta, Canada.

Hedengren, J.D., K.V. Allsford and J. Ramlal (2007). Moving horizon estimation and control for an industrial gas phase polymeriza- tion reactor. In: Proceedings of the American Control Conference. New York, NY.

Liebman, M.J., T.F. Edgar and L.S. Lasdon (1992). Efficient data reconciliation and estimation for dynamic processes using nonlinear programming techniques. Computers and Chemical Engineering 16, 963-986.

Marquardt, W. (2001). Nonlinear model reduction for optimization based control of transient chemical processes. In: Proc. CPC VI. number 326 In: AIChE Symposium Series. pp. 3060.

Moraal, P.E. and J.W. Grizzle (1995). Observer design for nonlinear systems with discretetime measurements. IEEE Transactions on Automatic Control 40(3), 395-404.

Qin, S.J. and T.A. Badgwell (2000). Nonlinear Model Predictive Control. Chap. An overview of nonlinear model predictive control applications, pp. 369-392. Birkhäuser Verlag. Boston, MA.

Ray, W.H. (1972). On the mathematical modeling of polymerization reactors. J. Macromol. Sci.-Revs. Macromol. Chem. C8(1), 1-56.

Russo, L.P. and R.E. Young (1999). Movinghorizon state estimation applied to an industrial polymerization process. In: In Proc. American Control Conf.. San Diego, CA. pp. 1129-1133.

Soderstrom, T.A., T.F. Edgar, L.P. Russo and R.E. Young (2000). Industrial application of a large-scale dynamic data reconciliation strategy. Industrial and Engineering Chemistry Research 39, 1683-1693.

Young, R.E. (2001). Evolution of an industrial nonlinear model controller. In: Proc. of the CPC VI. Tucson, AZ. 